\title{
EXTRAÇÃO E CARACTERIZAÇÃO DE ÁCIDOS HÚMICOS DE TRÊS CARVÕES DO SUL DO BRASIL
}

\author{
F. de SOUZA, P. KROTH, G. PAROLIN, S. R. BRAGANÇA \\ Universidade Federal do Rio Grande do Sul, Departamento de Materiais \\ E-mail para contato: fabihflor@gmail.com
}

\begin{abstract}
RESUMO - Os ácidos húmicos, maiores componentes da matéria orgânica e utilizados principalmente na produção de fertilizantes organominerais, podem ser extraídos de diferentes matérias-primas, como resíduos de animais, lodo de esgoto, deposições geológicas de origem orgânica como turfa e linhito oxidado. Isto é devido ao grande número de grupos funcionais contidos em suas moléculas, sua natureza polimérica e sua estabilidade química. Diferentes agentes oxidantes podem ser utilizados, seguidos de extração alcalina para obtenção dos ácidos húmicos. Estes, por sua vez podem variar muito em composição e funcionalidade de acordo com seu material de origem. Neste trabalho foram estudados três carvões do sul do Brasil: dois ao natural e um beneficiado. O objetivo deste trabalho foi obter rendimentos destes carvões de baixo poder calorífico com relação à extração de ácidos húmicos, bem como realizar a caracterização do produto gerado.
\end{abstract}

\section{INTRODUÇÃO}

As substâncias húmicas ( $\mathrm{SH}$ ) são os maiores componentes da matéria orgânica natural no solo e na água bem como em depósitos orgânicos geológicos como sedimentos de lagos, turfa, carvões de baixo rank e xistos. Elas são misturas complexas e heterogêneas de materiais polidispersos, formadas por reações químicas e bioquímicas durante a degradação e a transformação de resíduos de plantas e de micro-organismos (IHSS). As SH são divididas em ácido fúlvico (AF), ácido húmico (AH) e humina $(\mathrm{Hu})$, baseadas na sua solubilidade (CLASEN et al, 1998). Hu é a fração insolúvel em meio alcalino; $\mathrm{AH}$ é a fração escura extraída geralmente em meio alcalino e insolúvel em meio ácido diluído; AF é a fração colorida alcalino-solúvel que se mantém em solução após a remoção dos $\mathrm{AH}$ por acidificação (IHSS). Na natureza a humificação é um processo gradativo que leva milhares de anos, dando origem às substâncias húmicas $(\mathrm{SH})$ com diferentes características estruturais. Suas propriedades estão relacionadas com a complexação, transporte e biodisponibilidade de metais, conservação do solo, interação com pesticidas, etc (Freitas, 2002).

Os AH do carvão podem ser definidos como substâncias escuras derivadas dos carvões, às quais são solúveis em álcalis, mas insolúveis em ácidos. Elas ocorrem naturalmente em linhitos e carvões de baixo poder calorífico, mas pouco ou nenhum material solúvel em álcali está presente em carvões betuminosos (Skhonde et al., 2006). A quantidade de AH no carvão depende do grau de maturidade do carvão, bem como a natureza do processo de carbonização envolvido na sua formação (Stevenson, 
1994 citado por Skhonde et al., 2006). Nestes, a estrutura e bioquímica de formação, ainda pouco conhecidas, dependem do estágio de degradação da matéria orgânica, da mistura original dos organismos que lhes deram origem, das condições químicas, físicas e bioquímicas de decomposição, do processo de extração do sedimento e da atividade química e bioquímica ocorrida no meio ambiente (Clasen et al., 1998).

Os AH do carvão contêm carbono, hidrogênio, oxigênio e usualmente pequenas quantidades de enxofre e nitrogênio. Tratam-se de misturas complexas, que podem conter moléculas relativamente pequenas (ácidos himatomelânicos), além de suas largas moléculas polidispersas, e apresentam grande caráter aromático (Lawson; Stewart, 1989).

O objetivo deste trabalho foi obter rendimentos destes carvões de baixo poder calorífico com relação à extração de ácidos húmicos, bem como realizar a caracterização do produto gerado.

\section{MATERIAL E MÉTODOS}

Neste trabalho foram utilizados três carvões de baixo poder calorífico provenientes da região sul do Brasil: um carvão da Mina de Candiota-RS (CCA), um carvão de Minas do Leão-RS (CML), e um carvão da Carbonífera Cambuí-PR (CCC). Os carvões CCA e CML foram utilizados ao natural, e o carvão CCC sofreu um processo de beneficiamento para remoção das cinzas.

A Tabela 1 mostra o grau de umidade e o teor de cinzas dos carvões utilizados.

Tabela 1 - Umidade e cinzas dos carvões

\begin{tabular}{|c|c|c|}
\hline & Umidade (\%) & Cinzas*(\%) \\
\hline CCA & 1,65 & 53,64 \\
\hline CCC & 1,47 & 8,5 \\
\hline CML & 2,09 & 48,53 \\
\hline
\end{tabular}

*em base seca

Os carvões foram secos a $60{ }^{\circ} \mathrm{C}$, moídos e totalmente passados em peneira de abertura $250 \mu \mathrm{m}$. A extração dos $\mathrm{AH}$ foi realizada com $\mathrm{NaOH}$ 0,5mol/L em uma proporção 1:10 (carvão:extrator), submetido à agitação por 3h. Em seguida a suspensão foi centrifugada separando o sobrenadante, ao qual acidificou-se com $\mathrm{HCl}$ até $\mathrm{pH} \sim 2$, com agitação constante. A esta solução permitiu-se repouso de $24 \mathrm{~h}$ e após, centrifugou-se novamente. Ao precipitado realizou-se a purificação com solução $\mathrm{HCl} / \mathrm{HF}$ seguida de agitação por $2 \mathrm{~h}$. Após nova centrifugação, os $\mathrm{AH}$ obtidos foram lavados com água deionizada e secos em estufa a $60{ }^{\circ} \mathrm{C}$ por $24 \mathrm{~h}$, permitindo os cálculos de rendimento, e análises de caracterização.

Ao carvão CCA, realizou-se adicional e comparativamente uma extração dos AH utilizando uma etapa anterior de ataque ácido com solução $\mathrm{HCl}$ 0,5mol/L, a fim de avaliar a diferença de rendimento e caracterização resultante da remoção das cinzas. Todas as extrações foram realizadas em triplicata. 


\section{9 a 22 de outubro de 2014 \\ Florianópolis/SC}

Os cálculos de umidade foram realizados em estufa a $110{ }^{\circ} \mathrm{C}$ por $24 \mathrm{~h}$. As cinzas dos carvões foram calculadas utilizando o peso antes e após calcinação a $1000{ }^{\circ} \mathrm{C}$ por $6 \mathrm{~h}$, com taxa de aquecimento de $5{ }^{\circ} \mathrm{C} / \mathrm{min}$. As cinzas dos ácidos húmicos foram calculadas utilizando o peso antes e após calcinação a $800^{\circ} \mathrm{C}$ por $2 \mathrm{~h}$, com taxa de aquecimento de $5^{\circ} \mathrm{C} / \mathrm{min}$.

A Análise em espectroscopia de UV-Vis foi realizada medindo-se a absorbância em $445 \mathrm{~nm}$ e $665 \mathrm{~nm}$, após diluição de $4 \mathrm{mg}$ de ácido húmico em solução de bicarbonato de sódio 0,05 M. A espectroscopia de IV foi realizada utilizando pastilhas de $\mathrm{AH}$ com $\mathrm{KBr}$.

As amostras de carvão foram submetidas à extração alcalina com $\mathrm{NaOH}$. $\mathrm{Na}$ centrifugação, o precipitado é a Humina (Hu), que é descartada no processo. Já o sobrenadante, de coloração marromescura e com $\mathrm{pH} \sim 14,0$ contém as substâncias húmicas $(\mathrm{SH})$. Este líquido foi acidificado a um $\mathrm{pH} \sim 2$ com solução de $\mathrm{HCl}$, e a agitação seguida de repouso permitiu a separação de duas fases: uma fase líquida contendo ácidos fúlvicos (AF); e uma fase sólida contendo ácidos húmicos (AH). Os ácidos húmicos têm uma coloração preta intensa, e aspecto bastante viscoso.

A agitação do carvão com $\mathrm{NaOH}$ permite a separação entre $\mathrm{Hu}$ e $\mathrm{SH}$, já que estas últimas são solúveis em solução alcalina. A etapa de acidificação serve para separar os constituintes das SH: AF e AH. Como os AH são insolúveis em pH ácido, estes precipitam, e então torna-se possível esta separação das fases.

\section{RESULTADOS E DISCUSSÃO}

A Tabela 2 apresenta os rendimentos das extrações de AH com carvões CCA, CCC e CML. Os carvões brasileiros apresentam baixo rank, e grande quantidade de cinzas (Tabela 1). Por esta razão esperou-se obter baixos valores de rendimento na extração dos AH. De fato, observa-se na Tabela 2 que os rendimentos estiveram entre 0,37-1,4 \%. Estes valores são baixos em uma primeira análise, e levando em conta a quantidade inicial de carvão utilizado $(80 \mathrm{~g})$. Porém, ao se pensar em uma produção de $\mathrm{AH}$ em larga escala, este valor fica mais satisfatório, principalmente pelo fato de o carvão mineral brasileiro possuir um preço atraente.

Tabela 2 - Rendimento médio dos AH, umidade e cinzas

\begin{tabular}{|c|c|c|c|c|}
\hline $\begin{array}{c}\text { Ácido } \\
\text { húmico }\end{array}$ & $\begin{array}{c}\text { Rendimento médio** } \\
(\%)\end{array}$ & Desvio padrão & Umidade (\%) & Cinzas*** (\%) \\
\hline AH CCC3 & 0,52 & $\pm 0,16$ & 1,59 & 1,25 \\
\hline AH CML2 & 0,37 & $\pm 0,20$ & 4,96 & 5,97 \\
\hline AH CCA6 & 1,4 & $\pm 0,62$ & 10,12 & 8,5 \\
\hline AH CCA3 & 1,15 & $\pm 0,15$ & 10,77 & 10,33 \\
\hline
\end{tabular}

*AH CCA6: AH resultante do carvão CCA; AH CCC3: AH resultante do carvão CCC; AH CML2: AH resultante do carvão CML; AH CCA3: resultante do carvão CCA, mas que sofreu lavagem ácida com $\mathrm{HCl}$ previamente à extração. **em base seca e livre de cinzas. ***em base seca.

Os cálculos de rendimento foram realizados em base seca e isenta de cinzas, e as extrações 


\section{9 a 22 de outubro de 2014 \\ Florianópolis/SC}

foram feitas em triplicata. A segunda e terceira coluna da Tabela 2 mostram, respectivamente, os rendimentos médios e o desvio padrão das análises. Resultados similares de rendimento para um carvão da Jazida de Candiota minerado em 1995 foram encontrados por Clasen et al. (1998).

O carvão CCA foi o que apresentou um maior rendimento médio de $\mathrm{AH}$, porém neste carvão há maior quantidade de cinzas, e estas possivelmente não foram totalmente eliminadas na purificação com solução $\mathrm{HCl} / \mathrm{HF}$ e podem estar presentes como impurezas. Este fato pode ser verificado na Tabela 2 para AH CCA6 e AH CCA3, com 8,5\% e 10,33\% de cinzas, respectivamente. Por outro lado, o carvão CCC, ao qual esperava-se obter maior rendimento devido à quantidade muito baixa de materiais inorgânicos presentes (Tabela 1) apresentou rendimento bastante inferior ao CCA, porém, devido à pequena quantidade de cinzas presentes, acredita-se que se trata de um $\mathrm{AH}$ com maior pureza (Tabela 2). O carvão CML apresentou rendimento inferior entre os carvões estudados (Tabela 2). Este fato demonstra a necessidade de se adequar a extração de $\mathrm{AH}$ a condições específicas para cada matéria-prima, otimizando a metodologia. Fatores como temperatura de extração, tempo de agitação, relação carvão:extrator entre outros, podem influenciar no rendimento final de ácidos húmicos.

A fim de verificar a influência das cinzas presentes no carvão no rendimento dos $\mathrm{AH}$, realizaram-se duas lavagens ácidas no carvão CCA com solução de $\mathrm{HCl}$ 0,5 mol/L, antes da extração alcalina. Cada lavagem sofreu agitação por $2 \mathrm{~h}$ e repouso de $24 \mathrm{~h}$ seguido de centrifugação. $\mathrm{O}$ ataque ácido tem por objetivo remover carbonatos e metais do carvão; então o que se espera é que grande quantidade de sustâncias inorgânicas sejam solubilizadas e removidas antes da extração alcalina dos AH. Esta extração também foi realizada em triplicata, e é apresentada na Tabela 2 (AH CCA3). A partir dos dados encontrados, verifica-se que para o carvão CCA, os rendimentos de AH não variaram muito devido à lavagem ácida com $\mathrm{HCl}$. Este fato sugere que a etapa de purificação com solução $\mathrm{HCl} / \mathrm{HF}$ está sendo eficiente (e suficiente) no sentido da remoção de materiais inorgânicos. A pequena diferença no teor de cinzas dos AH do carvão candiota sustenta esta ideia (Tabela 2). Assim, em uma primeira análise, pode-se dispensar a etapa de lavagem ácida para o carvão Candiota, fato que gera economia de reagentes e de tempo de extração.

Os ácidos húmicos foram caracterizados quanto à composição química por FTIR. Ao AH CCA3 procedeu-se também a caracterização por espectroscopia de UV-Vis. No UV-Vis mediram-se as absorbâncias em comprimento de onda de $445 \mathrm{~nm}$ e $665 \mathrm{~nm}$, respectivamente fornecendo os valores de E4 e E6. A razão E4/E6 é muito utilizada para estimar a aromaticidade e humificação dos AH. A absorbância medida a $465 \mathrm{~nm}$ expressa a presença de ácidos húmicos formados em um estágio inicial de humificação, e a absorbância a $665 \mathrm{~nm}$ expressa a presença de ácidos húmicos formados na matéria orgânica bem humificada (Fong; Seng; Mat, 2007a). Para AH CCA3, a razão E4/E6 encontrada foi de 4,2, valor que está de acordo com a literatura (Garcia et al., 2003). Segundo Stevenson (1982), espera-se encontrar valores E4/E6 <5 para ácidos húmicos.

A Figura 1 mostra o espectro de FTIR para as amostras de ácido húmico extraídas dos carvões CCA, CCC e CML. O espectro dos AH extraídos mostrou um perfil bastante semelhante nos principais picos para todas as amostras analisadas e está coerente com o espectro de ácidos húmicos extraídos de carvões do sul do Brasil, de alto e baixo rank (Dick et al., 2002); além de carvão natural 
de outros países (Fong et al., 2006; Skhonde et al., 2006) e de carvão vegetal produzido por Eucaliptus spp. (Trompowsky et al., 2005). Os espectros, segundo Poppi e Talamoni (1992) apresentam bandas largas, com as regiões de absorção de cada grupo funcional indefinidas, comuns nos espectros de IV das SH, o que pode ser atribuído à extensiva superposição de absorções individuais.

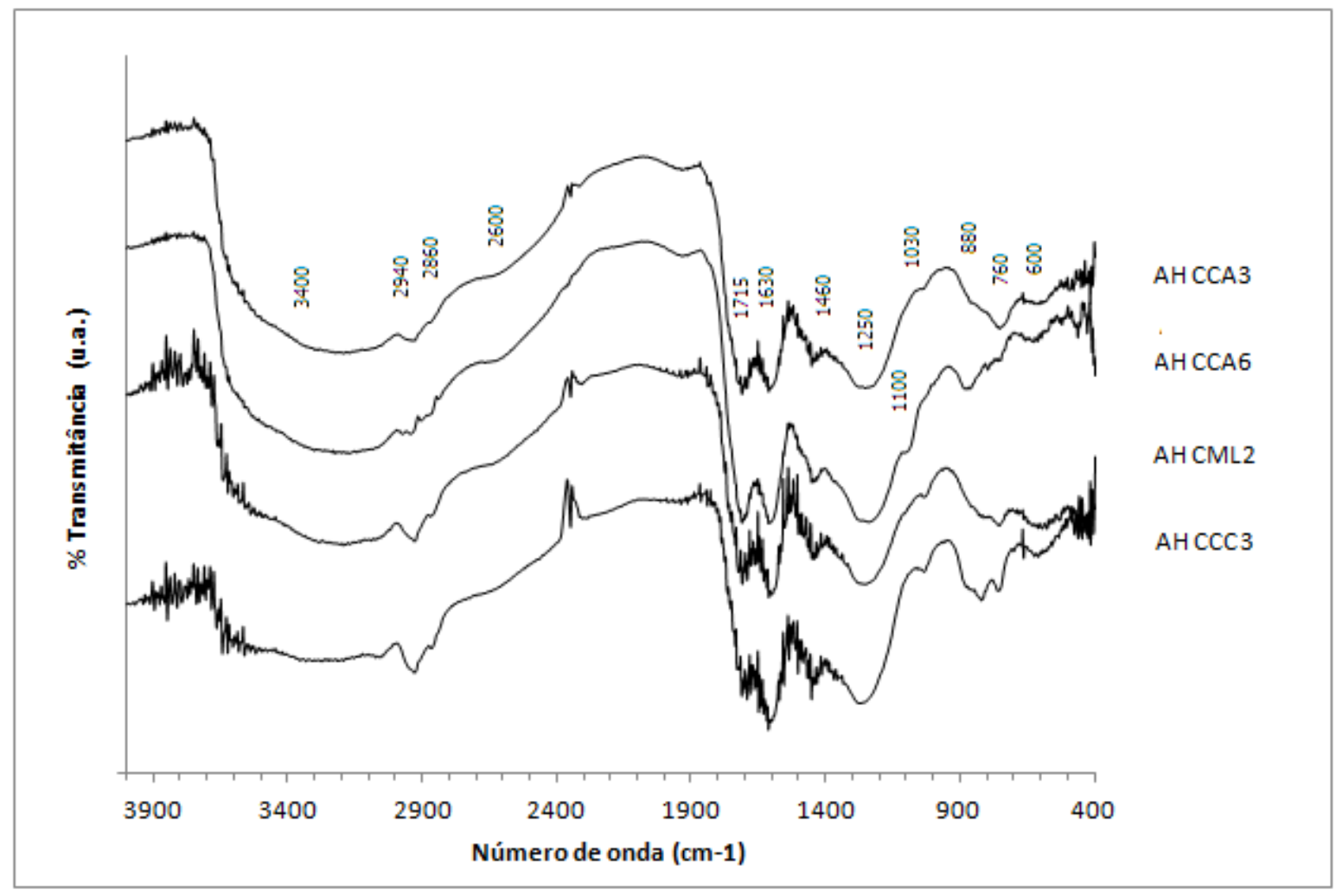

Figura 1 - FTIR para amostras de AH.

Dentre os principais picos observados no espectro IV, destacam-se: a) uma banda larga próxima a $3400 \mathrm{~cm}^{-1}$, resultado do estiramento $\mathrm{O}-\mathrm{H}$ de fenóis e álcoois além de água, presente como impureza (Fong et al., 2006); b) um par de bandas em $2940 \mathrm{~cm}^{-1}$ e em $2860 \mathrm{~cm}^{-1}$ que representam, respectivamente, o estiramento assimétrico e simétrico do $\mathrm{C}-\mathrm{H}$ alifático $\left(-\mathrm{CH}_{2}\right.$, $\left.\mathrm{CH}_{3}\right)$; c) um ombro em $2600 \mathrm{~cm}^{-1}$, é indicativo de grupos carboxílicos $(\mathrm{COOH})$ ligados através de ligações de hidrogênio (Trompowsky et al., 2005); d) Uma banda forte em $\sim 1715 \mathrm{~cm}^{-1}$ e um pico forte e bem definido em $1250 \mathrm{~cm}^{-1}$ são evidência da presença de grupos carboxílicos. $\mathrm{O}$ primeiro é atribuído ao estiramento $\mathrm{C}=\mathrm{O}$ de grupos carboxila e outros grupos carbonil (ex. cetonas). O segundo é atribuído ao estiramento C-O e deformação O-H de COOH, e estiramento C-O de fenóis, aromáticos, ésteres e aril-éteres (Santos e Camargo, 1999; Trompowsky et al., 2005); e) Uma banda forte e bem definida em $\sim 1630 \mathrm{~cm}^{-1}$, atribuída ao estiramento $\mathrm{C}=\mathrm{C}$ de aromáticos e íons carboxilato ( $\left.\mathrm{COO}^{-}\right)$. Porém, bandas entre 1690-1635, atribuídas a ligações 


\section{9 a 22 de outubro de 2014 \\ Florianópolis/SC}

duplas de alcenos conjugados e a $\mathrm{C}=\mathrm{O}$ de quinonas, podem absorver em frequências menores quando unidos por ligação de hidrogênio (Poppi; Talamoni, 1992; Fong et al., 2006).

Além destes, outros picos estão presentes no espectro, em menor intensidade. A banda em $1460 \mathrm{~cm}^{-1}$ representa o estiramento C-H alifático. Uma banda discreta entre $3077-3030 \mathrm{~cm}^{-1}$, característica do estiramento $\mathrm{C}-\mathrm{H}$ de aromáticos, está presente embora em alguns casos possa estar encoberta pela banda de estiramento do O-H (Poppi; Talamoni, 1992). Bandas entre 1100 e $1000 \mathrm{~cm}^{-1}$ são atribuídas a estruturas do tipo polissacarídeos (ligação $\mathrm{C}-\mathrm{O}$ ) e estiramento $\mathrm{Si}-\mathrm{O}$ $\left(1030 \mathrm{~cm}^{-1}\right)$ (Dick et al., 2002; Fong et al., 2006). Bandas com intensidade moderada entre 900$860 \mathrm{~cm}^{-1}$ são atribuídas ao anel benzênico com um ou dois átomos de hidrogênio não substituídos (Fong et al., 2007). Já as bandas entre $540-530 \mathrm{~cm}^{-1} \mathrm{e} 480-470 \mathrm{~cm}^{-1}$ são devido aos componentes minerais presentes na amostra de $\mathrm{AH}$. Os ácidos húmicos podem diferir significantemente com respeito aos seus constituintes inorgânicos, e esta variação pode ser devido a diferenças no processo de extração e purificação (Fong et al., 2006).

De acordo com Stevenson (1982), uma banda afiada próxima a $2315 \mathrm{~cm}^{-1}$ é devido ao $\mathrm{CO}_{2}$ aprisionado na matriz de $\mathrm{KBr}$, aparentemente proveniente da descarboxilação de grupos $\mathrm{COOH}$.

O AH CCA6 apresentou bandas menos intensas em 2940-2860 $\mathrm{cm}^{-1}$, e picos melhor resolvidos em 1715 e $1630 \mathrm{~cm}^{-1}$, e em $1460 \mathrm{~cm}^{-1}$. O AH CCC3 apresentou bandas mais intensas em 2940-2860 $\mathrm{cm}^{-1}$, o que sugere a presença de alifáticos. Porém, para este AH também há forte caráter aromático, como se pode perceber pela redução da banda em $1715 \mathrm{~cm}^{-1}$ e aumento da banda em $1630 \mathrm{~cm}^{-1}$ e pela banda em $3030 \mathrm{~cm}^{-1}$ melhor definida para AH CCC3, comparado com os demais AH. Todas as amostras apresentaram bandas características de absorção de anéis aromáticos, como pode ser visto pelas absorções abaixo de $900 \mathrm{~cm}^{-1}$, e acima de $3000 \mathrm{~cm}^{-1}$. A ausência de uma banda distinta em $3030 \mathrm{~cm}^{-1}$ pode ser explicada pela extensiva substituição do anel, além de estar mascarada pela forte absorção devido aos grupos $\mathrm{OH}$ com ligações de hidrogênio, que podem se estender em torno de $3600 \mathrm{~cm}^{-1}$ até $2900 \mathrm{~cm}^{-1}$ (Stevenson, 1982).

Todas as amostras de $\mathrm{AH}$ apresentaram banda pequena de ligação Si-O $\left(1030 \mathrm{~cm}^{-1}\right)$, evidenciando que nem toda sílica é diluída pelo $\mathrm{NaOH}$ utilizado na extração. Porém, segundo Fong et al (2006), este pico é inapropriado para caracterizar grupos atômicos já que uma pequena quantidade de silicatos pode induzir forte absorção.

Com relação à região inferior, dos constituintes inorgânicos, percebe-se que estas são mais destacadas para os AH provenientes dos carvões CCA e CML, e menos destacada para o AH do carvão CCC. Este fato está de acordo com as cinzas encontradas para estes AH (Tabela 2), e coerentes com a quantidade inicial de cinzas dos carvões (Tabela 1).

Comparando-se as amostras AH CCA3 e AH CCA6, respectivamente com e sem tratamento ácido previamente à extração, não se verificou diferenças significativas no espectro IV. Apenas verificou-se em AH CCA6 uma banda em $1100 \mathrm{~cm}^{-1}$ que não foi observada em AH CCA3. Assim, a lavagem com $\mathrm{HCl}$ pode estar sendo mais eficiente na remoção de polissacarídeos. 


\section{9 a 22 de outubro de 2014 \\ Florianópolis/SC}

\section{CONCLUSÕES}

O carvão CCA foi o que apresentou maior rendimento de ácidos húmicos (>1\%), quando comparado com outro carvão de baixo rank e com um carvão beneficiado. Porém, o ácido húmico correspondente apresentou maior quantidade de contaminantes inorgânicos.

O carvão beneficiado com remoção de grande parte de material inorgânico e pequeno teor de cinzas (CCC) gerou rendimento de ácidos húmicos inferior ao carvão de grande teor de cinzas (CCA) utilizando o mesmo processo. Assim, torna-se necessário estudar a extração caso a caso e otimizar de acordo com a matéria-prima utilizada.

O valor E4/E6 para AH CCA3 está de acordo com os valores encontrados na literatura para ácidos húmicos.

Os espectros FTIR mostram similaridade para os diferentes ácidos húmicos extraídos, apresentando basicamente os mesmos grupos funcionais.

O teor de cinzas nos ácidos húmicos está de acordo com seu teor no carvão, e gera uma diferença discreta no espectro IV.

Com relação à utilização de lavagem ácida anterior à extração dos ácidos húmicos, percebe-se que, utilizando esta metodologia, a lavagem ácida não é atraente levando-se em consideração o tempo empregado neste processo. Os rendimentos de extração foram muito similares, e os espectros de IV também foram muito próximos. A principal diferença no espectro IV foi a presença de uma banda a $1100 \mathrm{~cm}^{-1}$ em AH CCA6 que não foi vista em AH CCA3, e sugere que a lavagem ácida esteja sendo mais eficiente no sentido da remoção de contaminantes do tipo polissacarídeos. O teor de cinzas nestes $\mathrm{AH}$ mostra que há pouca diferença quando se emprega a lavagem ácida.

\section{REFERÊNCIAS}

CLASEN, H. A. C.; LESSA, R. T.; KAEMMERER, M.; KOETZ, P. R. Ácidos Húmicos e Fúlvicos do carvão da jazida de Candiota. Rev. Bras. de Agrociência, v. 4, n 1, p. 35-40, JanAbril 1998.

CHEN, Y.; SENESI, N.; SCHNITZER, M. Information Provided on Humic Substances By E4/E6 Ratios. Soil Sci. Soc. Am. J, v. 41, n² 2, p. 352-358, 1 march 1977.

DICK, D. P.; MANGRICH, A. S.; MENEZES, S. M. C.; PEREIRA, B. F. Chemical and spectroscopical characterization of humic acids from two south brazilian coals of different ranks. J. Braz. Chem. Soc, v. 13, n 2, p. 177-182, 2002.

FONG, S. S.; SENG, L.; MAJRI, N. Bt; MAT, H. Bt. A comparative evaluation on the oxidative approaches for extraction of humic acids from low rank coal of Mukah, Sarawak. J. Braz. Chem. Soc, v. 18, $\mathrm{n}^{\circ} 1$, p. 34-40, 2007. 
FONG, S. S.; SENG, L.; CHONG, W. N.; ASING, J.; NOR, M. F. b Md; PAUZAN, A. S. bt Mohd. Characterization of the coal derived humic acids from Mukah, Sarawak as soil conditioner. J. Braz. Chem. Soc, v. 17, n 3, p. 582-587, 2006.

FONG, S. S.; SENG, L. Bt; MAT, H. Reuse of nitric acid in the oxidative pretreatment step for preparation of humic acids from low rank coal of Mukah, Sarawak. J. Braz. Chem. Soc., v. 18, ${ }^{\circ}$ 1, 41-46, 2007a.

FREITAS, Josias C. de; SANTANA, Genilson P. Mineralogia e caracterização dos ácidos húmicos e fúlvicos de uma topossequência da reserva Adolpho Ducke (Manaus-AM). Revista Ciência, p. 84-91, 2002.

GARCIA, S. S.; MORO, D. B.; SILVA, R. M. da; JABLONSKI, A. Efeito da oxidação do carvão de baixo rank na produção de ácidos húmicos. V Encontro Brasileiro de Substâncias Húmicas. Grupo Brasileiro da IHSS. Curitiba-PR, p. 134-135, 2003.

IHSS - International Humic Substances Society. Disponível em: <http://www.humicsubstances.org/whatarehs.html >. Acesso em: 03 dez. 13.

LAWSON, G. J.; STEWART, D. Coal Humic Acids. Cap. 23. In: Humic Substances II In search of structure. John Willey \& Sons Ltda., 1989.

POPPI, N. R.; TALAMONI, J. Estudo dos ácidos húmico e fúlvico, extraídos de solos, por espectroscopia de infravermelho. Quím. Nova, v. 15, n4, 1992.

SANTOS, Gabriel de Araújo; CAMARGO, Flávio A. de O. (editores). Fundamentos da matéria orgânica do solo: ecossistemas tropicais e subtropicais. 1. ed. Porto Alegre: Genesis, 1999. 580 p.: il. Capítulos.

SKHONDE, M. P.; HEROD, A. A.; VAN DER WALT, T. J.; TSATSI, W. L.; MOKOENA, K. The effect of thermal treatment on the compositional structure of humic acids extracted from South African bituminous coal. Int. J. Miner. Process, 81, p. 51-57, 2006.

STEVENSON, F. J. Humus Chemistry: Genesis, composition, reactions. $2^{\circ}$ ed. John Wiley \& Sons, New York, 1982. 496 p.

TROMPOWSKY, P. M.; BENITES, V. de M.; MADARI, B. E.; PIMENTA, A. S.; HOCKADAY, W. C.; HATCHER, P. G. Charecterization of humic like substances obtained by chemical oxidation of eucalyptus charcoal. Org. Chem., v. 36, p. 1480-1489, 2005. 